\section{Macular hole surgery with silicone oil}

\section{Abstract}

Purpose Macular hole surgery usually involves gas tamponade which necessitates a variable period of postoperative posturing in a face-down position. A number of patients find this difficult or impossible for medical or social reasons. This study reviews our experience of macular hole surgery using silicone oil tamponade with no restriction of posturing.

Methods Sixteen consecutive cases of macular hole (stage III/IV) surgery where silicone oil was used for tamponade were reviewed. Group A patients were unable for medical or social reasons to maintain a facedown posture and Group B patients had previous failed surgery with gas tamponade. Results Anatomical closure of hole was achieved in $81 \%(13 / 16)$ of all cases and $88 \%$ (7/8) of cases with no previous surgery (Group A) with a mean follow-up of 7.5 months (range 4-24 months). Mean visual improvement $\left(\log _{\text {MAR }}\right.$ value of visual acuity) for Group A and Group B were 0.41 and 0.03 , respectively.

Conclusions The success rate of macular hole surgery using silicone oil as tamponade, in cases with no previous surgery, is comparable to that achieved when gas is used as tamponade. Although good anatomical success can be achieved in cases with previous failed surgery, the visual outcome is less rewarding. Silicone oil is an alternative to gas tamponade for macular hole surgery in patients who are unable or unwilling to posture. However, it has to be borne in mind that silicone oil tamponade is not without risks.

Eye (2002) 16, 121-125. DOI: 10.1038/

sj/EYE/6700029

Keywords: macular hole; macular hole surgery; posture; silicone oil

\section{Introduction}

The first report of successful macular hole surgery was published by Kelly and Wendel
V Kumar, S Banerjee, AV Loo, AB Callear and MT Benson

in $1991 .^{1}$ In recent years, a number of authors have attributed increased success rate of surgery to the use of healing adjuvants (autologus serum, transforming growth factor $\beta$, platelets or thrombin), the initial stage and duration of macular hole. ${ }^{2-8}$

The surgical technique common to the above studies necessitates postoperative facedown posturing in order to achieve effective tamponade of the macular hole. Indeed, there is evidence to suggest that longer duration of intraocular gas tamponade may have a favourable effect on the outcome of macular hole surgery..$^{9,10}$

Although two studies have reported comparable results with no face-down posture $^{11}$ and four days of posture ${ }^{12}$ postoperatively, most studies advocate strict face-down posturing for at least one week after surgery as this is believed to be an important factor in closure of the hole. Because of this, macular hole surgery has been restricted to patients who are able to comply with the postoperative face-down posturing.

A number of patients, however, are unable to posture because of positioning difficulties due to neck, back, spine, chest, other diseases or social reasons. This study reviews our experience of macular hole surgery using silicone oil tamponade without posturing.

\section{Materials and methods}

Sixteen consecutive cases of macular hole surgery using silicone oil for tamponade were reviewed. Data from preoperative assessment, surgery and postoperative follow-up were collected. The main outcome measures were best corrected pre- and postoperative visual acuity, anatomical status of the macular hole and complications. Patients were refracted independently pre- and postoperatively and visual acuity was measured with the Snellen chart.

A three-port pars plana vitrectomy was performed in all patients. For stage III macular holes, the posterior hyaloid was detached by active aspiration over the
Birmingham and Midland Eye Centre

Birmingham, UK

Correspondence:

MT Benson

Birmingham \& Midland Eye Centre

City Hospital, Dudley Road Birmingham B18 7QH, UK

Tel: 01215543801

Fax: 01215076791

E-mail: markbenson@ talk21.com

The above authors do not have proprietary interest in any of the products mentioned in this paper

Presented at: The Royal Society of Medicine, London, UK, June 1999 
posterior hyaloid using the vitrectomy probe and simultaneous retraction of the probe. Vitrectomy was then completed as far out to the periphery as possible. Visible epiretinal membranes were removed. In our unit it has not been standard practice to search for and peel epiretinal membranes although there have been reports to suggest that this is associated with improved results. A careful examination of the peripheral fundus was performed and any retinal breaks were treated with cryopexy. Fluid-air exchange was followed by a 10-min pause to allow residual fluid to accumulate posteriorly. This fluid was then removed with passive aspiration directly over the macular hole. In all eyes $0.1 \mathrm{ml}$ of autologus serum was injected over the posterior pole. Air-silicone oil (1300 centistokes) exchange was carried out to complete the surgery. Postoperatively patients had no restriction of posturing. Removal of silicone oil was performed after a minimum of 4 weeks following surgery.

The macular holes were graded as closed only if the edges were completely sealed and not visible ie flat closed (FC). They were graded as flat open (FO) if the edge of the hole was still visible and elevated open (EO) if any part of the open hole was elevated. ${ }^{13}$

\section{Results}

All 16 cases had idiopathic full thickness stage 3 or 4 macular holes. There were two distinct groups of patients. Group A $(8 / 16)$ had primary surgery because patients in this group were unable or unwilling to posture. Group B (8/16) were patients in whom primary surgery with gas tamponade had failed and in whom poor compliance with postoperative posturing was thought to be a major cause of this failure.

The mean age of patients for the two groups was 68.3 years (range 58-76 years) for Group A and 68.9 years (range 65-73 years) for Group B (Table 1). Fourteen $(88 \%)$ patients were female. There were eight (56\%) stage III and eight (44\%) stage IV macular holes. The mean duration of symptoms (ie from first

Table 1 Patient summary

\begin{tabular}{|c|c|c|}
\hline Sex & $\begin{array}{l}\text { female }-14 \\
\text { male }-2\end{array}$ & $\begin{array}{l}(88 \%) \\
(12 \%)\end{array}$ \\
\hline Mean age & 68.6 years & (range $58-76$ years) \\
\hline Stage of FTMH & $\begin{array}{l}\text { Stage III }-9 \\
\text { Stage IV }-7\end{array}$ & $\begin{array}{l}(56 \%) \\
(44 \%)\end{array}$ \\
\hline \multicolumn{3}{|l|}{$\begin{array}{l}\text { Mean duration of } \\
\text { symptoms }\end{array}$} \\
\hline Group A & 10.6 months & (range $4-18$ months) \\
\hline Group B & 20.3 months & (range $10-24$ months) \\
\hline
\end{tabular}

FTMH, full thickness macular hole.
Table 2 Post operative anatomical results

\begin{tabular}{lcccc}
\hline & Closed (FC) & Flat open (FO) & Open (EO) & Total \\
\hline Group A & $7(88 \%)$ & $1(12 \%)$ & 0 & 8 \\
Group B & $6(75 \%)$ & $2(25 \%)$ & 0 & 8 \\
Total & $13(81 \%)$ & $3(19 \%)$ & 0 & 16 \\
\hline
\end{tabular}

Group A, primary surgery.

Group B, secondary surgery following previous failed surgery.

symptom to time of surgery with silicone oil) was 10.6 months (range 4-18 months) for Group A and 20.3 months (range 10-24 months) for Group B. At the time of primary surgery the mean duration of symptoms for Group B was 14.9 months (range 4-22 months). All patients had a minimum of 4 months follow-up postoperatively with a mean of 7.5 months (range 4-24 months). The mean for removal of silicone oil was 13 weeks postoperatively (range of 4-30 weeks).

Macular holes were closed (FC) in $81 \%(13 / 16)$ of all cases and $88 \%(7 / 8)$ of cases with no previous surgery (Group A). The three remaining cases had flat open (FO) holes postoperatively (Table 2). Two holes (one from each group) which were initially closed (FC) reopened (EO) following removal of silicone oil. Hence the long-term closure rate was $69 \%(11 / 16)$ for all cases with a mean follow-up of 7.5 months (range 4-24 months).

Preoperative visual acuity was $6 / 36$ or worse in all cases. Postoperatively best visual acuity was $6 / 9$ part in Group A and 6/18 part in Group B. Visual acuity improved by two lines or more in $63 \%(5 / 8)$ of cases in Group A and 25\% (2/8) of cases in Group B (Figures 1 and 2 ). Mean visual improvement ( $\log _{\text {MAR }}$ value of visual acuity) for Group A and Group B was

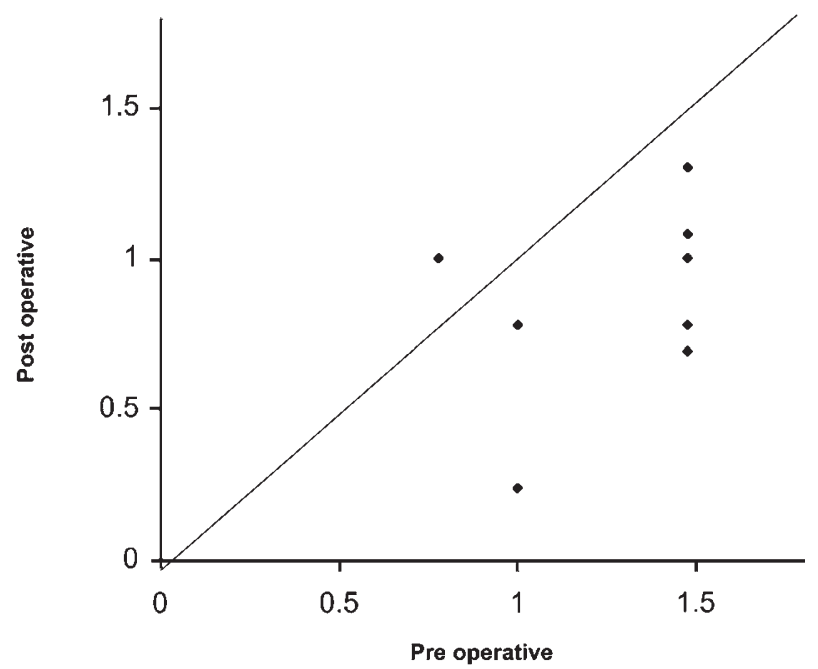

Figure 1 Pre- and postoperative $\log _{\mathrm{MAR}}$ equivalent of visual acuity for Group A. 


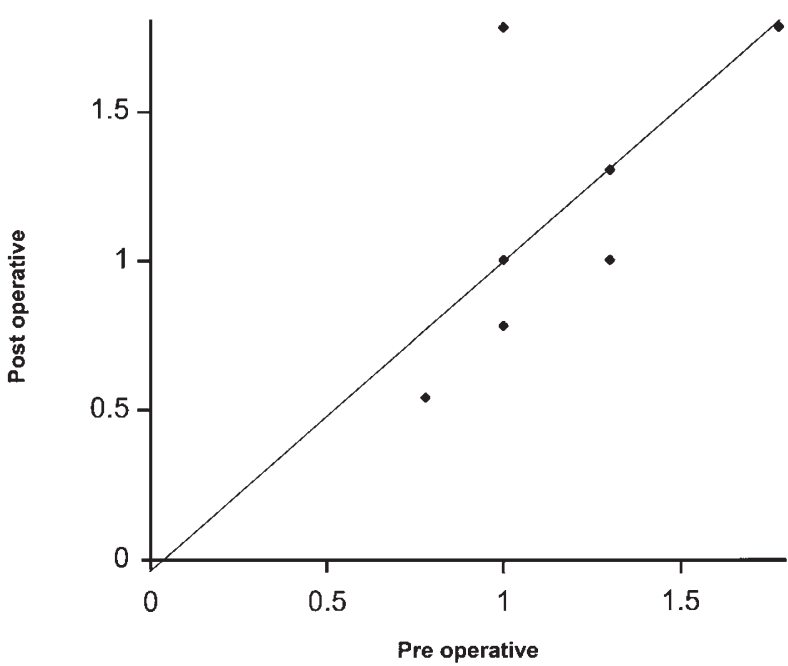

Figure 2 Pre- and postoperative $\log _{\mathrm{MAR}}$ equivalent of visual acuity for Group B.

0.41 and 0.03 respectively ( 0.22 for all cases). For cases with closed holes (FC) mean $\log _{\text {MAR }}$ value of visual acuity improved by 0.35 to 0.87 .

At the time of surgery $94 \%(15 / 16)$ of eyes were phakic. Postoperatively progression of nuclear sclerosis was noted in all phakic eyes. In three cases removal of silicone oil was combined with cataract extraction by phacoemulsification and intraocular lens implant. In two cases retinal detachment occurred following removal of silicone oil. This may have resulted as a complication of either the primary macular hole surgery or at the time of removal of silicone oil. One case was associated with reopening of the macular hole. Both retinas were successfully reattached. Other complications included lens touch $(1 / 16)$, entry site breaks (2/16), re-opening of macular hole following removal of oil $(2 / 16)$ and raised intraocular pressure postoperatively (4/16). Intraocular pressure in all four cases settled following removal of oil.

Table $3 \log _{\text {MAR }}$ equivalent of Snellen visual acuity

\begin{tabular}{lc} 
Snellen acuity & $\log _{\text {MAR }}$ Equivalent \\
\hline $1 / 60$ & 1.78 \\
$3 / 60$ & 1.30 \\
$6 / 60$ & 1.00 \\
$6 / 36$ & 0.78 \\
$6 / 24$ & 0.60 \\
$6 / 18$ & 0.48 \\
$6 / 12$ & 0.30 \\
$6 / 9$ & 0.18 \\
$6 / 6$ & 0.00 \\
\hline
\end{tabular}

\section{Discussion}

The ultimate goal of macular hole surgery is visual improvement. Since improvement in postoperative vision has been shown to be linked to anatomical closure of the hole, surgeons and researchers have concentrated their efforts on modifying their surgical technique to achieve a better hole closure rate.

The first reported series of surgery (vitrectomy and gas tamponade) for stage III/IV macular hole achieved a $58 \%$ closure rate. ${ }^{1}$ The same authors subsequently reported a success rate of $89 \%$ with increasing experience. The benefits of surgery for stage III/IV macular holes were established by a multicentred randomised clinical trial which obtained a closure rate of $69 \% .^{7}$ Since the first reports, a number of authors have reported their improved success rates with modification in surgical techniques including peeling of epiretinal membranes and the use of adjuvants. ${ }^{2-6,9,10,14}$ It is also likely that success rates are modified by case selection.

However, they have relied on the use of gas tamponade and the need for the patients to posture face down postoperatively. Surgery has therefore not been an option for patients who are unable to posture because of positioning difficulties due to neck, back, spine, chest, other diseases or social reasons such as with those patients who live on their own. For an agerelated disease this represents a significant number of patients. In our study with the use of silicone oil as tamponade there was no restriction of posturing. The initial anatomical closure rate of $88 \%$ for primary surgery (Group A) is comparable to those reported with gas tamponade for stage III / IV holes (5891\%). ${ }^{13,7,14}$ The closure rate for repeat surgery was $75 \%$ in our series. Previously reported results of anatomical closure following reoperation with gas tamponade vary from $73-91 \%$. 3,5,15,16

The mean visual improvement ( $\log _{\text {MAR }}$ equivalent of visual acuity, Table 3) in Group A patients was 0.41. Snellen visual acuity improved by two lines or more in $63 \%(5 / 8)$ of cases in the same group. This compares favourably with previously reported results for primary surgery with gas tamponade for stage III / IV macular holes (42-60\%). ${ }^{1,3,17}$ In cases with previous failed surgery (Group B) only 25\% (2/8) of cases improved by two or more lines. Others have reported two or more lines of improvement in $25-75 \%$ of cases. ${ }^{1,3,16,17}$ The mean visual improvement for Group B was negligible when compared with Group A (0.03 vs 0.41 on $\log _{\text {MAR }}$ equivalent). Previous reports have also shown poorer visual outcome for reoperation cases although the difference has been less marked than in our series. ${ }^{3,18}$ Possible explanations for this difference 
in our series could be a selected population with poor ability for reapposition of retinal photoreceptors, as indicated by failure of primary surgery, poor preoperative visual acuity, poor capacity for functional recovery and larger holes. Clinically, macular pigment changes were more commonly seen postoperatively in Group B patients. All these factors may be related to the longer duration between onset of symptoms and hole closure in Group B patients. In our study the mean duration of symptoms to first operation for Group B patients was 14.9 months (range 4-22 months) followed by an average delay of 5.4 months in reoperation time.

A recent series using silicone oil tamponade reported closure rate of $83 \%$ and mean visual improvement ( $\log _{\text {MAR }}$ equivalent of visual acuity) of 0.24 for stage III/IV macular holes. ${ }^{18}$ These results are comparable to our series ( $81 \%$ and 0.22$)$. The poorer pre- and postoperative vision in our series is likely to be related to the longer hole duration (mean of 7.1 vs 15.4 months). A mean follow-up of 7.5 months is relatively short for assessing visual acuity in patients with macular hole surgery and may underestimate the final visual improvement.

Macular hole surgery with silicone oil tamponade is an option worth considering in cases with previous failed surgery (Group B), particularly in the presence of fellow eye pathology and if there are difficulties with posturing. This is because good anatomical closure rate can be achieved for these patients and there is substantial variability in visual improvement among eyes with successful closure of macular hole. Perhaps in future if we are able to predict the visual outcome for individual cases one can be more selective regarding candidates for reoperation.

The success rate of macular hole surgery using silicone oil as a tamponade in cases with no previous surgery would appear to be comparable to that achieved when gas tamponade is used. We recommend silicone oil as an alternative to gas tamponade for primary macular hole surgery in patients who are unable or unwilling to posture. Silicone oil tamponade is not without its risks-the necessity for a second operation, cataract formation and risk of glaucoma and silicone toxicity. However, in the group of patients where gas tamponade with posturing would not be possible and hence surgery was deferred, silicone oil with no posturing may be a viable alternative. The patients would need to be counselled preoperatively regarding the risks and likelihood of surgery not being as successful as with gas tamponade. In our series the rate of retinal detachment is quite high $(12.5 \%)$, compared to other series as is the rate of late reopening (12.5\%). Careful analysis of these cases failed to reveal any causes for this.

This small study demonstrates the need for a controlled study with greater numbers for statistical comparison of results.

\section{References}

1 Kelly NE, Wendel RT. Vitreous surgery for idiopathic macular holes: results of a pilot study. Arch Ophthalmol 1991; 109: 654-659.

2 Glaser BM, Michels RG, Kupperman BD et al. Transforming growth factor $\beta 2$ for the treatment of full thickness macular holes: a prospective randomized study. Ophthalmology 1992; 99: 1162-1173.

3 Minihan M, Goggin M, Cleary PE. Surgical management of macular holes: results using gas tamponade alone, or in combination with autologus platelet concentrate, or transforming growth factor $\beta 2$. Br J Ophthalmol 1997; 81: 1073-1079.

4 Christmas NJ, Skolik SA, Howard MA et al. Treatment of retinal breaks with autologus serum in experimental model. Ophthalmology 1995; 102: 263-271.

5 Liggett PE, Skolik SA, Hario B et al. Human autologus serum for the treatment of full thickness macular holes: a preliminary study. Ophthalmology 1995; 102: 1071-1076.

6 Kim JW, Freeman WR, Azen SP et al. Prospective randomized trial of vitrectomy or observation for stage 2 macular holes: vitrectomy for macular hole study group. Am J Ophthalmol 1996; 121: 605-614.

7 Freeman WR, Azen SP, Kim JW et al. Vitrectomy for the treatment of full thickness stage 3 or 4 macular holes. Results of a multicentral randomized clinical trial. The vitrectomy for treatment of macular hole study group. Arch Ophthalmol 1997; 115: 11-21.

8 Willis AW, Garcia-Cosio JF. Macular hole surgery: comparison of longstanding versus recent macular holes. Ophthalmology 1996; 103: 1811-1814.

9 Thompson JT, Glaser BM, Sjaarda RN et al. Effects of intraocular bubble duration in the treatment of macular holes by vitrectomy and transforming growth factor $\beta 2$. Ophthalmology 1994; 101: 1195-2000.

10 Thompson JT, Smiddy WE, Glaser BM et al. Intraocular tamponade duration and success of macular hole surgery. Retina 1996; 16: 373-382.

11 Tornambe PE, Poliner LS, Grote K. Macular hole surgery without face-down posturing. A pilot study. Retina 1997; 17: 179-185.

12 Park DW, Sipperley JO, Sneed SR, Dugel PU, Jacobsen J. Macular hole surgery with internal-limiting membrane peeling and intravenous air. Ophthalmology 1999; 106: 1392-1397; discussion 1397-8.

13 Tornambe PE, Poliner LS, Cohen RG. Definition of macular hole surgery endpoints. Retina 1998; 18: 286-287.

14 Smiddy WE, Glaser BM, Thompson JT et al. Transforming growth factor $\beta 2$ significantly enhances the ability to flatten the rim of subretinal fluid surrounding macular holes. Retina 1993; 13: 296-301.

15 Smiddy WE, Sjaarda RN, Glaser BM et al. Reoperation after failed macular hole surgery. Retina 1996; 16: 13-18.

16 Christmas NJ, Smiddy WE, Flynn HW Jr. Reopening of macular holes after initially successful repair. Ophthalmology 1998; 105: 1835-1838. 
17 Thompson JT, Sjaarda RN, Lansing MB. The results of vitreous surgery for chronic macular holes. Retina 1997; 17: 493-501.
18 Goldbaum MH, McCuen BW 2nd, Hanneken AM et al. Silicone oil tamponade to seal macular holes without position restrictions. Ophthalmology 1998; 105: 2140-2147. 